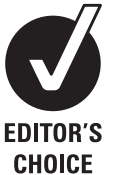

${ }^{1}$ Center for Injury Research and Prevention at The Children's Hospital of Philadelphia, Philadelphia, Pennsylvania, USA ${ }^{2}$ Department of Forensic Medicine, Monash University, Melbourne, Victoria, Australia ${ }^{3}$ Accident Research Centre, Monash University, Melbourne, Victoria, Australia

${ }^{4}$ Chinese Centers for Disease Control and Prevention, Beijing China

${ }^{5}$ Division of General Pediatrics, Department of Pediatrics, The Children's Hospital of Philadelphia, Philadelphia, Pennsylvania, USA ${ }^{6}$ Leonard Davis Institute for Health Economics, University of Pennsylvania, Philadelphia, Pennsylvania, USA

\section{Correspondence to}

Dr Flaura K Winston, c/o Center for Injury Research and

Prevention, The Children's Hospital of Philadelphia, 3535

Market Street, 11th floor, Philadelphia, PA 19104, USA; flaura@mail.med.upenn.edu

Accepted 10 April 2010

\title{
Cultural translation: acceptability and efficacy of a US-based injury prevention intervention in China
}

\author{
Danielle Erkoboni, ${ }^{1}$ Joan Ozanne-Smith, ${ }^{2,3}$ Cao Rouxiang, ${ }^{4}$ Flaura K Winston ${ }^{1,5,6}$
}

\section{ABSTRACT}

Background Increased belt-positioning booster seat (BPB) awareness and access have led to increased use in the USA. Although transportation in Beijing is rapidly becoming 'motorised', Beijing's population has limited awareness of or access to BPBs.

Objective To explore the efficacy and acceptability of using a US-developed BPB use promotion intervention in Beijing.

Design Methods were adapted from a previously executed US-based study involving parents of 3-8-year old children. Focus groups (five groups, 71 participants) elicited behavioural antecedents to BPB use and reactions to video interventions promoting BPB use: a Chinese-produced instructional video and an Englishlanguage (dubbed into Mandarin) video that delivered concrete, theoretically driven messages through a personal story. Immediately after the focus groups, participants were provided with education and a free BPB. Participants were contacted 6 weeks later via telephone about use, knowledge and attitudes.

Results Chinese parents saw safety as the most important benefit of BPB use; lack of accurate knowledge about and access to BPBs were parents' most prevalent barriers. Chinese participants described the videos as persuasive and instructional. At 6 weeks, participants remembered the messages of the English-language video, and reported BPB use increased from a baseline of $15.5 \%$ to $85.5 \%$.

Conclusions This study shows the possibility of exporting US-designed prevention interventions dubbed into Mandarin without the need to alter their original context (in this case, an African American family in a US setting) into a Chinese context. Successful cultural translation involved ensuring that the behavioural antecedents targeted in the intervention (eg, barriers and benefits) were of relevance to the Chinese population.

\section{INTRODUCTION}

Globally, road traffic injuries are the leading cause of death and acquired disability for children and accounted for $24 \%$ of all injury-related deaths of children aged 0-14 in 2004. ${ }^{1}$ Although use of safety belts for children in vehicles provides a level of protection, appropriate restraint use along with riding in the rear seat have been found to decrease the risk of injury by $71 \%$ for children between the ages of 4 and 12 years over safety belts alone. ${ }^{2}$ As a result, the US National Highway Traffic Safety Administration recommends belt-positioning booster seat (BPB) use with safety belts for children aged $4-8 .^{3}$

In China, a middle class is emerging with young families who have acquired motor vehicles, but low child restraint system/booster seat use puts the children at risk of injury in motor vehicle crashes.
The Beijing Traffic Management Bureau has estimated that the vehicle fleet on Beijing roadways increases at a rate of nearly 1500 new automobiles per day. ${ }^{4}$ With this increasing motorisation, the World Health Organization estimates that, overall, more than 45000 road users are injured daily on China's roads. ${ }^{5}$ Intervention programmes are currently in place in Beijing to increase awareness about the importance of adult safety belts; however, the benefits of appropriate child occupant protection are not very well known. A recent survey of 30000 vehicles in two Chinese cities identified no children using child restraints and only $2.5 \%$ of 1315 children under 8 years of age using adult seat belts. ${ }^{6}$

In the USA, a multi-pronged approach that improved awareness about and access to BPBs resulted in high $\mathrm{BPB}$ use within this age group. ${ }^{7-12}$ One particular intervention was developed and tested according to behavioural theory. ${ }^{13} 14$ The programme theory on which the intervention was developed included target constructs that promoted intentions to use booster seats: knowledge about $\mathrm{BPBs}$ (eg, specific injuries prevented with use); selfefficacy about use (eg, demonstrating how to use); dispelling negative beliefs (eg, misperceptions about lack of safety benefit from not being anchored to the seat); and overcoming barriers to access to BPBs. The intervention involved two phases: watching a video followed by receipt of a free $\mathrm{BPB}$ and education on its use. Surveys administered before and after the video showed the effectiveness of this intervention in changing attitudes and intentions about booster seat use among the participants.

\section{Study purpose}

The purpose of this study was to explore whether a booster seat promotion intervention, grounded in behavioural theory, but originally developed for and evaluated by a US population, ${ }^{13}$ would be efficacious for and acceptable to a Chinese population. Further, this study explored whether the mixed qualitative/quantitative methods used in the US study could be adapted to the Chinese context. It was hypothesised that this programme (videobased education and provision of a free BPB with training in its use), grounded in behaviour change theory, would be able to translate across cultural boundaries if the target populations possessed common perceived benefits, disadvantages, facilitators, and barriers to $\mathrm{BPB}$ use.

\section{Theoretical framework and original study methodology}

To address the need to promote BPB use in China, an elicitation and intervention study was designed 
on the basis of a theoretical model that draws largely from the Integrative Model of Behaviour Change, ${ }^{15}$ which incorporates concepts from the Theory of Reasoned Action, ${ }^{16}$ the Theory of Planned Behaviour, ${ }^{17}$ and Social Cognitive Theory. ${ }^{18}$ At the model's core, BPB use behaviour is preceded by a positive intention to perform the behaviour, which, in turn, depends on addressing target constructs including perceived benefits, facilitators, and barriers to performing that behaviour.

\section{METHODS}

Using the theoretical framework and core methods from the previous US-based study, this study used mixed qualitative and quantitative methods to (1) identify factors that influence parents' current child restraint behaviours and intentions for future use, and (2) test interventions that address these factors as a means to promote positive intention towards BPB use behaviours and actual self-reported behaviour.

\section{Recruitment and sample}

Parents were recruited for the study through kindergartens and elementary schools in the Chaoyang, HaiDian, and ChangPing districts of Beijing by members of the research team from the Division of School Health in the Beijing Centers for Disease Control (CDC). Eligible parents were those who owned a car and had children between the ages of 3 and 8 years enrolled in a Beijing kindergarten or elementary school. Parents who did not meet these study criteria were excluded. Once a school agreed to participate, parents were informed about the study through previously scheduled parent-teacher meetings. Interested parents were then screened for eligibility and invited to attend. The design of this study was approved by the institutional review boards at Monash University and The Children's Hospital of Philadelphia and by the Beijing CDC.

All study materials from the US-based study were translated into Mandarin and back-translated to ensure accuracy. This included all written survey materials, the focus group facilitator guide, and the follow-up telephone survey instrument. All focus groups and interviews were conducted in Mandarin. In addition, the US-designed video intervention 'No Regrets' was dubbed into Mandarin. No additional changes were made to the video (eg, changing the ethnicity of the video's subjects, etc) at the request of the Chinese investigators.

\section{Description of videos evaluated}

The videos shown in Beijing included a Mandarin-language knowledge-only video developed by a Chinese automotive safety organisation and a Mandarin-dubbed, English-language knowledge-plus-motivation video ('No Regrets').

\section{Knowledge-only video}

The Chinese-designed video provided facts around the benefits of child safety restraints, paired with graphic visual demonstrations of the benefits of booster seat use and the effects of non-use. This video was designed for a Chinese audience with Chinese actors and was recorded in Mandarin.

\section{Knowledge-plus-motivation video}

This English-language video entitled 'No Regrets', (http://www. research.chop.edu/programs/carseat/boosterseatvideos.php) was designed for a US audience, recorded in English, and featured an African-American narrator. For this study, the video was dubbed into Mandarin with Mandarin subtitles. Although this video included educational components on $\mathrm{BPB}$ use, its content went beyond knowledge by presenting the information in a way that addresses multiple target constructs and an actionable emotion-avoiding regret by preventing a child's injury through use of $\mathrm{BPB}$. The video delivered the targeted messages as a personal narrative, and other best-practice communication techniques were used to increase the likelihood that the injury prevention messages were understandable, memorable, and effective in changing thoughts and behaviour. ${ }^{19}$

\section{Description of study protocol}

The study protocol involved both intervention and data collection (qualitative and quantitative). In order to measure potential changes in participant knowledge, perceptions, attitudes, and intentions related to child restraint and BPBs, three unique, paper-based surveys were administered during each focus group. All participants were given a survey at the appropriate time during the focus groups and allowed adequate time and support to answer all questions.

\section{Baseline survey}

Baseline measures of restraint knowledge, attitudes, and use and demographic data were gathered in a short survey instrument administered before the start of discussion.

\section{Collection of qualitative data on target constructs}

Initial focus group discussion elicited parents' perceived benefits, disadvantages, facilitators, and barriers to BPB use.

\section{Collection of qualitative data on reactions to videos}

As part of the focus group, parents were shown the two videos detailed above. Discussion around each video followed the presentation of the video. In all groups, the knowledge-only video was shown first, with the knowledge-plus-motivation video ('No Regrets') shown second.

After the presentation of both videos, further focus group discussion ascertained parents' reactions to the two targeted intervention videos. In this discussion, participants were asked to recommend ways in which the videos could be improved to convey messages around BPB use to other parents. Transcripts were supplemented by collection of field notes by trained researchers.

\section{Post-discussion survey}

This second survey instrument measured immediate post-video changes in behavioural beliefs, and assessed whether the videos engaged the participants, addressed their barriers and threats, and promoted positive intentions to use BPBs. This survey was administered after participants had viewed and discussed the interventions, and contained many items repeated from the baseline survey.

Collection of qualitative data after BPB provision and demonstration Each focus group was followed by provision of a BPB (the only incentive provided for participation) and education and demonstration on BPB use to each parent. Trained observers recorded comments and questions from parent participants.

\section{Six-week follow-up telephone survey}

Longer-term self-reported behaviour change and recall of interventions was measured by a telephone follow-up assessment 6 weeks after the focus group discussion. All telephone followup calls were conducted by research team members fluent in Mandarin. Participants were contacted at their homes using information that they provided at study enrolment. Attempts 
were made to contact all participants; participants who could not be reached after three attempts were excluded. No additional incentives were provided for participation outside of the free $\mathrm{BPB}$ provided at the time of the focus group.

In the 6-week follow-up survey, adoption of and adherence to $\mathrm{BPB}$ use behaviour was measured according to the 'stages of change' of Prochaska and DiClemente ${ }^{20}$ : precontemplation, contemplation, preparation, action, and maintenance of use, and termination of non-use. A five-item, progressive, non-threatening algorithm was used to measure self-reported BPB use for the participants' children. Participants were first asked whether they had thought about using the BPB; if so, whether they had tried it; if so, whether they use it regularly; and if so, whether they have told others to use it (a measure of extinguishing of the non-use behaviour). Questions were modelled after previously validated question wording developed by Littell and Girven. ${ }^{21}$

\section{Data analysis}

All focus group discussions were taped, transcribed, and translated into English. Transcripts were coded by research staff on the basis of the themes of participants' perceived barriers, benefits, and threats relating to BPB use. In addition, parent responses to the video interventions were coded to qualitatively assess participant's response to the programmes. To supplement these transcripts, field notes were taken by researchers present during the discussion. A translator was present during the focus group for each English-speaking researcher.

Changes in attitudes and intentions to use a BPB were also assessed quantitatively through the information collection forms. For descriptive analyses of interval scale variables (eg, participant age and number of children), the mean, median, mode, and range were obtained. These variables were also categorised for further analysis. Frequencies were calculated for categorical variables.

\section{RESULTS \\ Sample}

A total of 71 parents participated in the eight focus groups conducted in Beijing, China. All parents completed the surveys administered both before and after the focus group discussion. Of this group, $89.9 \%(n=62)$ completed the follow-up interview administered by telephone 6 weeks after the initial focus group discussion.

The majority of parents who participated in this study were aged 31 to $35(56.5 \%)$, and married (98.6\%) (table 1). Most participants were employed outside the home (91.5\%), with $80.3 \%$ of those employed working full time.

\section{Qualitative focus group data}

\section{Parental beliefs and knowledge}

Chinese parents indicated in focus group discussions that their child's safety was very important and the most important benefit of BPB use, and that the most significant barrier to BPB use was lack of knowledge. While these parents claimed to have heard of or seen BPBs on occasion, they did not have concrete information on when and how to use a BPB for their children. They were also relatively unaware of the safety benefit of BPBs. Participants cited that, although seatbelts were effective for adults, they did not fit their children properly. Many parents believed the safest and most comfortable place for a child in the car is held on the lap of an adult.

\section{Parental reactions to videos}

Participants felt that both videos provided clear messages about the safety benefit of booster seats and that they had learned new
Table 1 Participant demographics

\begin{tabular}{lc}
\hline Characteristic & \% of sample $(\mathbf{n}=\mathbf{7 1})$ \\
\hline Age & \\
$26-30$ & 7.0 \\
$31-35$ & 54.9 \\
$36-40$ & 25.4 \\
$41-45$ & 7.0 \\
45 and older & 2.8 \\
Blank & 2.8 \\
Marital status & \\
Married & 98.6 \\
Living with partner & 0.0 \\
Single, never married & 0.0 \\
Separated & 0.0 \\
Divorced & 1.4 \\
Blank & 0.0 \\
Employed & \\
No & 8.5 \\
Yes & 91.5 \\
Work status of those employed & \\
Full-time & 80.3 \\
Part-time & 2.8 \\
Blank & 17.0 \\
Job type of those employed & \\
Medical & 12.5 \\
Administration/Office & 12.5 \\
Service & 37.5 \\
Other & 27.5 \\
Blank & 10.0 \\
\hline & \\
\hline
\end{tabular}

information (ie, that booster seats were designed for safety, rather than to simply increase a child's comfort in a car). The knowledge-only video did not generate discussion among the parents about beginning to use a BPB.

Despite the American setting and African-American characters depicted, Chinese participants showed immediate motivation to use a BPB after watching the US-developed knowledge-plus-motivation video ('No Regrets'). In each focus group, this video elicited discussion on how to make BPBs work in their lives. Discussion topics after this video frequently included the logistics of BPB use. While parents appreciated the information contained in the knowledge-only video, the coupling of knowledge with actionable emotions in the knowledge-plus-motivation video spoke to the parent's previously elicited target constructs and was cited as more effective in motivating them to change their behaviour.

\section{Parental reactions to BPB-use demonstration}

After each focus group, a child-passenger-safety technician demonstrated proper BPB use with a child in an actual car, and each parent was provided with a free BPB. Participants had concerns that, as the BPB was not tethered to their car's seat, it would fall forward even when held by a seatbelt. These concerns were reexpressed by many parents in the follow-up telephone interview.

\section{Quantitative survey data}

Before the focus group discussion, $15.5 \%$ of parents had used a booster seat for their child, all while travelling abroad with their families. After the discussion, $81.7 \%$ of parents reported BPB use intentions as at least somewhat likely to use a booster seat to restrain their child in the car in the next 2 months. Of those who participated in the follow-up survey 6 weeks after the focus group ( $\mathrm{n}=62), 85.5 \%$ reported using a BPB on their most recent trip with their child (table 2). 
The two videos together were credited by Chinese parents as the primary source of motivation toward BPB use. In addition to reported BPB use, the US-designed, knowledge-plus-motivation video 'No Regrets' was widely recalled at 6 weeks. Specifically, parents cited this video's narrator and the family's story in the telephone survey as continued reminders of the safety benefit of BPBs. In addition, $41.9 \%$ of respondents had let someone else know about the story in the video and the safety benefit of BPBs. When asked for additional comments, many parents noted that they would like the US-based video's message to be more widely available.

\section{CONCLUSIONS}

Results from this study show that: (1) a theoretically grounded, motivational intervention created in the USA and merely dubbed into Mandarin was acceptable and efficacious among Chinese parents; (2) a collaborative qualitative and behavioural research study informed by behavioural theory that follow-up protocols established in the USA could be executed in Beijing.

This study applied best practice in behavioural intervention development and evaluation and the use of a programme theory ${ }^{14}$ that allowed the successful cross-cultural translation. Firstly, the intervention was theoretically grounded in behavioural theory. Secondly, the protocol included multiple points of measurement. The intervention, which involved videos, discussion, education, and provision of a BPB, was designed to address elicited barriers and facilitators to BPB (eg, attitudes and beliefs) in a context of actionable emotion-avoiding regret. The efficacy of the intervention in the Chinese population, with no revision besides dubbing into Mandarin, likely stems from the fact that the US and Chinese populations shared similar barriers and facilitators. Differences in culture (eg, ethnicity of the family, setting of the video) were secondary to the message that addressed the barriers and facilitators.

Thirdly, the study design measured not only behavioural adoption but also changes in the perceived barriers (eg, attitudes and beliefs). For example, by collecting baseline attitudes, beliefs, and barriers both individually and within the focus group setting, the investigators were able to test the applicability of the underlying programme theory in the Chinese context. By so doing, if the intervention was found not to be efficacious, data would be available to revise both the theory and the resultant intervention. Further cultural translation in other contexts could adapt this methodology to assess baseline barriers to BPB use to determine whether this intervention can be applied elsewhere.

The methods used in this study translated smoothly into a Chinese context, with the need for very few adaptations. The Chinese study required direct translation of the US-study instruments and focus group moderator guide into Mandarin and training of the Chinese investigators in focus group methodology and data analysis. Completion of this translation, along with the planning and execution of the study was accomplished with the aid of an international collaboration that brought together experience in injury research and Chinese beliefs and practices. This collaboration was critical in executing this study across cultures.

In addition to demonstrating successful translation of the protocol, this study also highlights the possibility of exporting adapted US prevention initiatives to a Chinese context. "No Regrets', a video intervention designed within a US context, featured the story of an African-American parent, but was motivational to Chinese parents without the need for cultural adaptation (aside from dubbing into Mandarin). Parents were receptive to the clear, concrete message that used the actionable emotion of regret to highlight commonly held behavioural antecedents (beliefs about child safety), and provide knowledge around $\mathrm{BPBs}$ to remove barriers. As in the original US context, ${ }^{13}$ the video intervention delivered in the Chinese context during a focus group and coupled with distribution of BPBs was motivational and memorable.

The only difference noted among the two groups regarding reactions to the booster seat intervention was apprehension around the safety of the unattached booster seat expressed by the Chinese but not the US subjects. This difference may be partly attributed to the difference in the extent of general passenger safety messages between the two countries. Although much work remains, US drivers and passengers have been exposed to messages around booster seats and motor vehicle safety for several years. Although many campaigns have begun to introduce road traffic safety messages in China, much more work is needed to create a trust in vehicle safety measures in this and other rapidly motorising countries. ${ }^{22} 23$ Regardless, it will be important to address this concern in a wider distribution of this interventionvideo and additional educational/training opportunities.

Although a body of research exists on the need for cultural adaptation of health messages for distinct cultures within the USA, $^{24-26}$ very little work has explored the effectiveness of using available interventions across cultures internationally. While this research begins to explore the feasibility of this approach, it should be seen as a call to action to evaluate existing interventions and determine their ability to address public health issues emerging abroad without the time and expense of major adaptations.

\section{Study limitations and future work}

Whereas two focus group phases were conducted in the original US study, one condensed phase was held in the Chinese study. This change was made because of time constraints and feasibility. As a result, the focus group moderator guide for the Chinese study highlighted each of the topics covered in the US study, but went into less depth on certain chosen constructs. Aside from this change, the methods used in this Chinese study replicated those previously executed and evaluated in the USA.

The generalisability of the results is limited because of the methodology that used a convenience cross-sectional sample

Table 2 Current BPB use and intentions about future BPB use in a Beijing population

\begin{tabular}{ll}
\hline Question & $\%$ of sample \\
\hline Ever used a booster seat for your child* & $\mathrm{n}=71$ \\
No & 84.5 \\
Yes & 15.5 \\
Blank & 0.0 \\
Will use a booster seat in the next 2 & $\mathrm{n}=71$ \\
months $\dagger$ & \\
Very likely & 69.0 \\
Somewhat likely & 12.7 \\
Not sure & 2.8 \\
Somewhat unlikely & 1.4 \\
Very unlikely & 0.0 \\
Blank & 14.1 \\
Used a booster seat on most recent trip $\ddagger$ & $\mathrm{n}=62$ \\
No & 11.3 \\
Yes & 85.5 \\
Blank & 3.2 \\
\hline
\end{tabular}

*From questionnaire administered before focus group discussion.

†From questionnaire administered after focus group discussion.

$\neq$ From 6-week follow-up survey.

$\mathrm{BPB}$, belt-positioning booster seat. 
(chosen across socioeconomic status groups and districts of Beijing) of parents in focus groups. Although this study design allowed for a thorough discussion on a wide range of topics related to BPB use for parents of children living in Beijing, this study would need to be replicated or another design used in other areas of China to determine whether the broader Chinese population holds similar beliefs about BPB use and whether the intervention proves efficacious in that population. While this study provided insights into parental beliefs in Beijing, it may not be representative of the whole Chinese (or Beijing) population. It would be useful for additional focus groups to be conducted among a diverse sample of Chinese parents in different locations until saturation is reached for all groups.

The relative individual importance of the videos, the demonstration and the provision of free booster seats could not be determined from this study. Further research would be necessary to discern the effects of the individual components outside of the whole programme.

In addition, further research is required to explore the adaptation of the methodology to the cultural translation of other health-promotion interventions developed in the USA. Finally, future work should include implementation and evaluation of a large-scale media campaign to promote broader knowledge and acceptance of BPBs and improved access to reasonably priced BPBs.

\section{Implications}

This study demonstrates the possibility of exporting USdesigned prevention interventions dubbed into Mandarin without the need to alter their original context (in this case, an African-American family in a US setting) into a Chinese context. Successful cultural translation involved ensuring that

\section{What is already known on this subject}

- China is rapidly becoming motorised, with nearly 1500 new vehicles being registered daily in Beijing.

- Road traffic safety messages have begun to be introduced in China; however, much more work is needed to propagate these messages and create trust in in-vehicle safety devices and practices.

- One means to achieve this goal is to adapt messages and interventions proven effective in the USA to a Chinese context. Little work has explored the effectiveness of cultural translation of interventions designed for a US audience.

\section{What this study adds}

- This study shows the possibility of exporting US-designed interventions to a Chinese context when the intervention addresses commonly held behavioural antecedents.

- For effective intervention translation, the target and original populations should be evaluated and compared with regard to perceived benefits from, and barriers to, the target behaviour.

- The success of this individual intervention should be seen as a call to action to public health professionals to evaluate existing interventions and determine the existing interventions ability to address public health issues emerging abroad. the behavioural antecedents targeted in the intervention (eg, barriers and benefits) were of relevance to the Chinese target population.

Acknowledgements We acknowledge the Industrial Advisory Board Member companies of the Industry/University Cooperative Research Center, Center for Child Injury Prevention Studies (CChIPS), and the US National Science Foundation (NSF) for their financial support of this study and, specifically, Takata Corporation for the provision of booster seats to the participants of this study. We also thank the staff at The Beijing Centers for Disease Control, Beijing Consulting Group (specifically Edward Smith), The Center for Injury Research and Prevention at the Children's Hospital of Philadelphia and the Monash University Accident Research Centre for their help and support in the development and evaluation of this project. We also acknowledge the China Automobile Association for supplying its BPB video and its technical staff to demonstrate BPBs and their use.

Funding Centers for Child Injury Prevention Studies (CChIPS) and the National Science Foundation (NSF) provided the financial support that made this study possible.

Competing interests None.

Ethics approval This study was conducted with the approval of the Children's Hospital of Philadelphia, Monash University, and The Chinese Centers for Disease Control and Prevention.

Contributors All authors took part in the planning, conduct, and reporting of the work described in this article. This includes contribution to the project's conception and design, analysis and interpretation of data drafting, critical revision of the draft manuscript, and final approval of the revised content.

Provenance and peer review Not commissioned; externally peer reviewed.

\section{REFERENCES}

1. Peden M, Oyegbite K, Ozanne-Smith J, et al. eds. World report on child injury prevention. Geneva: World Health Organization and UNICEF, 2008.

2. Durbin DR, Chen I, Smith R, et al. Effects of seating position and appropriate restraint use on the risk of injury to children in motor vehicle crashes. Pediatrics 2005; 115:305-9.

3. National Highway Traffic Safety Administration. Improving the safety of olderchild passengers. (DOT HS 809 953). Washington, DC: U.S. Department of Transportation, National Highway Traffic Safety Administration, 2005.

4. Beijing Traffic Management Bureau. Beijing has more cars on roads. (Press release) Published online: 17 Feb 2009. http://210.75.211.252/publish/portal1/ tab165/info10441.htm (accessed 6 Apr 2009).

5. World Health Organization. World report on road traffic injury prevention, 2004 http://www.who.int/violence injury prevention/publications/road traffic/world report/en/index.html. (accessed 11 Jul 2008).

6. Routley V, Ozanne-Smith J, Li D, et al. China belting up or down? Seat belt wearing trends in Nanjing and Zhoushan. Accid Anal Prev 2008;40:1850-8.

7. Simpson EM, Moll EK, Kassam-Adams N, et al. Barriers to booster seat use and strategies to increase their use. Pediatrics 2002:110:729-36.

8. Rivara FP, Bennett E, Crispin B, et al. Booster seats for child passengers: lessons for increasing their use. Inj Prev 2001:7:210-13.

9. Weiss-Laxer NS, Mello MJ, Nolan PA. Evaluating the educational component of a hospital-based child passenger safety program. J Trauma 2009:67(1 Suppl): S30-3.

10. Ebel BE, Coronado GD, Thompson B, et al. Child passenger safety behaviors in latino communities. J Health Care Poor Underserved 2006:17;358-73.

11. Bracchitta KM. Factors influencing parental use of booster seats for their children J Clin Psychol Med Settings 2006:13;270-81.

12. National Highway Traffic Safety Administration. Identifying information that promotes belt-positioning booster use, volume l: summary and findings. (DOT HS 811 018). Washington, DC: U.S. Department of Transportation, National Highway Traffic Safety Administration, 2008.

13. Winston FK, Erkoboni D, Xie D. Identifying interventions that promote beltpositioning booster seat use for parents with low educational attainment. J Trauma 2007:63:S1-S10.

14. Winston FK, Jacobsohn L. A practical approach for applying best practices in behavioral interventions to injury prevention. Injury Prevention 2010;16:107-12.

15. Fishbein $\mathbf{T}$, Yzer M. Using theory to design effective health behavior interventions Commun Theory 2003;13:164-83.

16. Fishbein T, Ajzen I. Belief, attitude, intention, and behavior: an introduction to theory and research, 2nd edn. Reading. MA: Addison-Wesley Pub. Co, 1975

17. Ajzen I. The theory of planned behavior. Organ Behav Hum Decis Process 1991:50:179-211.

18. Bandura A. Social cognitive theory. In: Vasta R, ed. Six theories of child development: revised formulations and current issues. Greenwich, CT: JAI Press, 1992:1-60.

19. Heath C, Heath D. Made to stick: why some ideas survive and others die. New York Random House, 2007 
20. Prochaska J0, DiClemente CC. Stages and processes of self-change of smoking: Toward an integrative model of change. J Consult Clin Psychol 1983;51:390-5.

21. Littell JH, Girvin H. Stages of change: a critique. Behav Modif 2002;26:223-73.

22. Zhang W, Huang $Y$, Roetting $M$, et al. Driver's views and behaviors about safety in China - What do they NOT know about driving? Accid Anal Prev 2006;38:22-7.

23. Wang SY, Li YH, Chi GB, et al. Injury-related fatalities in China: an under-recognized public health problem. Lancet 2008;372:1765-73.
24. Chao CM. The central role of culture: working with Asian children and families. In Kaslow FW, ed. Comprehensive handbook of psychotherapy: Interpersonal/ humanistic/existential Vol. 3. New York: Wiley, 2002:35-58.

25. McDaniel SH, Lusterman DD, Philpot CL. Casebook for integrating family therapy: An ecosystemic approach. Washington, DC: American Psychological Association, 2001

26. McGoldrick M, Giordano J, Pearce JK. Ethnicity and family therapy, 2nd edn. New York: Guilford Press, 1996.

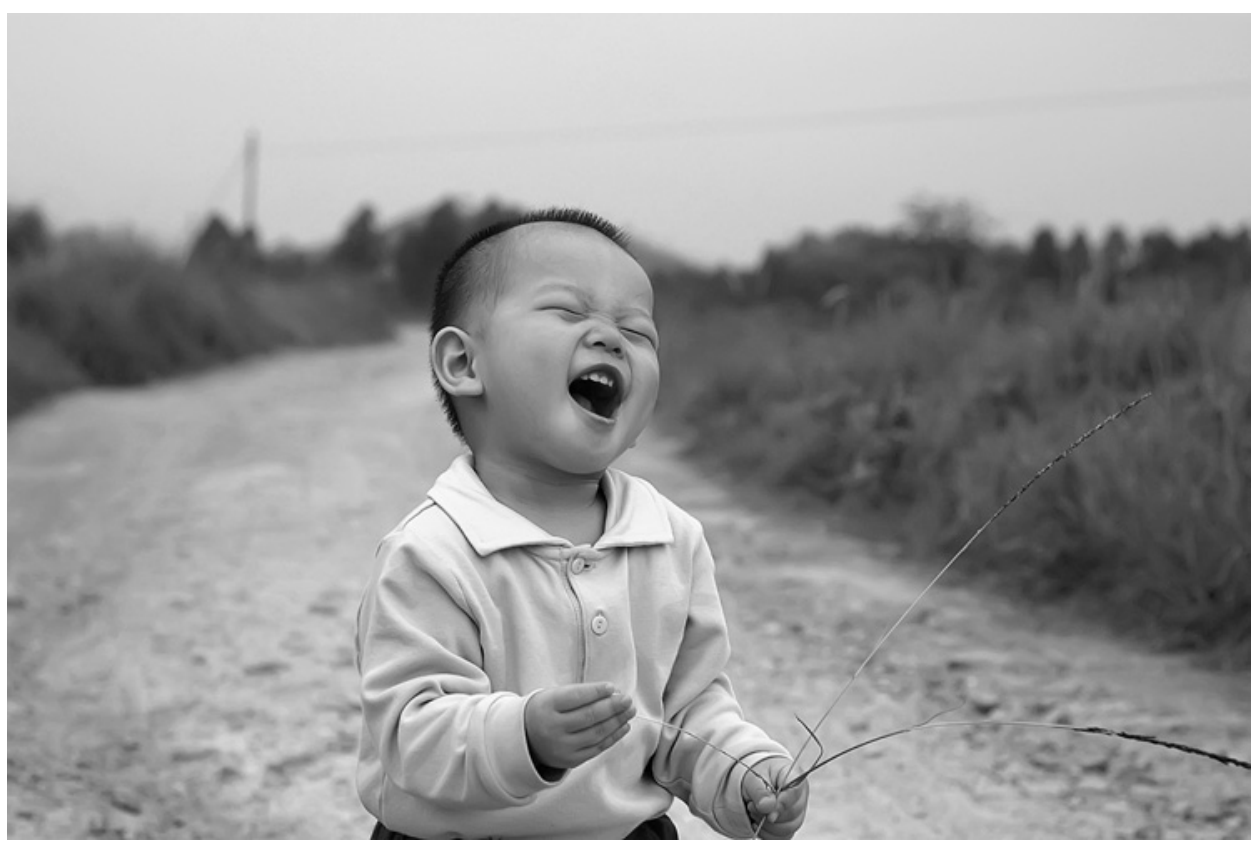

